\title{
SmartHeart CABG Edu
}

\section{Gabriele di GIAMMARCO ${ }^{\mathrm{a}}$, Tania di MASCIO ${ }^{\mathrm{b}}$, Michele di MAURO ${ }^{\mathrm{c}}$, Antonietta TARQUINIO ${ }^{\mathrm{d}}$, and Pierpaolo VITTORINI ${ }^{\mathrm{c}}$}

${ }^{a}$ Dep. of Neurosciences, imaging and clinical sciences, University of Chieti, Chieti, Italy

${ }^{\mathrm{b}}$ Dep. of Information Engineering, Computer Science and Mathematics, University of L'Aquila, L'Aquila, Italy

${ }^{\mathrm{c}}$ Dep. of Life, Health and Environmental Sciences, University of L'Aquila, L'Aquila, Italy

${ }^{\mathrm{d}}$ Cardiology Unit, AUSL Pescara, Pescara, Italy

KEYWORD

Pre-operative education; user experience; anxiety
Abstract

The paper reports on the SmartHeart CABG Edu Android app. The app was conceived to be an innovative and up-to-date tool for patient education, the first of its kind in the Italian context. In particular, the app was developed to provide educational material for patients about to undergo Coronary Artery Bypass Graft $(C A B G)$ surgery, a set of self-assessment tools concerning health status (i.e., BMI calculator, LDL cholesterol calculator and anxiety assessment tool) and usability questionnaires (i.e., SEQ and SUS). The paper initially describes the app, then reports on its evaluation, concerning both the app usability and the pre-operative anxiety, and ends by showing the improvements - derived from the usability evaluation - put into practice.

\section{Introduction}

Patient education - usually one of the most important responsibilities of nurses - is the process by which health professionals impart information to patients, their family members and/or their caregivers. During acute illness, patient education is usually health information given at bedside to help patients understand diagnosis and treatment and, therefore, enhance their adherence to clinical care. In the case of chronic illness, education helps the patient and the family members to increase knowledge about the disease, develop expertise in self-care and improve lifestyle by changing unhealthy behaviors (e.g., reducing the intake of fatty foods) and correcting the related risk factors (e.g., stop smoking).

In Coronary Artery Disease (CAD), education is essential for empowering patients in both the acute and the chronic phases of the disease, involving the patients in their plan of care in the former case and helping them being compliant to healthy behaviors, in the latter. However, CAD is a chronic disease: even after Coronary Artery Bypass Graft (CABG) surgery, the disease progression can only be slowed down. Pre-operative education facilitates the patients' understanding of the diagnosis and treatment of CAD and in adopting healthy lifestyles for improving outcomes. For those about to undergo CABG, patient education can be even more significant since these patients may experience great anxiety for a life-threatening intervention. Various studies have been conducted concerning pre-operative education of patients about to undergo CABG surgery. (Young et al., 2009) and (Black and Hawks, 2009) state that patients confronted with a life-threatening

Regular Issue

Vol 4 n.1

http://adcaij.usal.es
Advances in Distributed

Computing and Artificial Intelligence Journal 

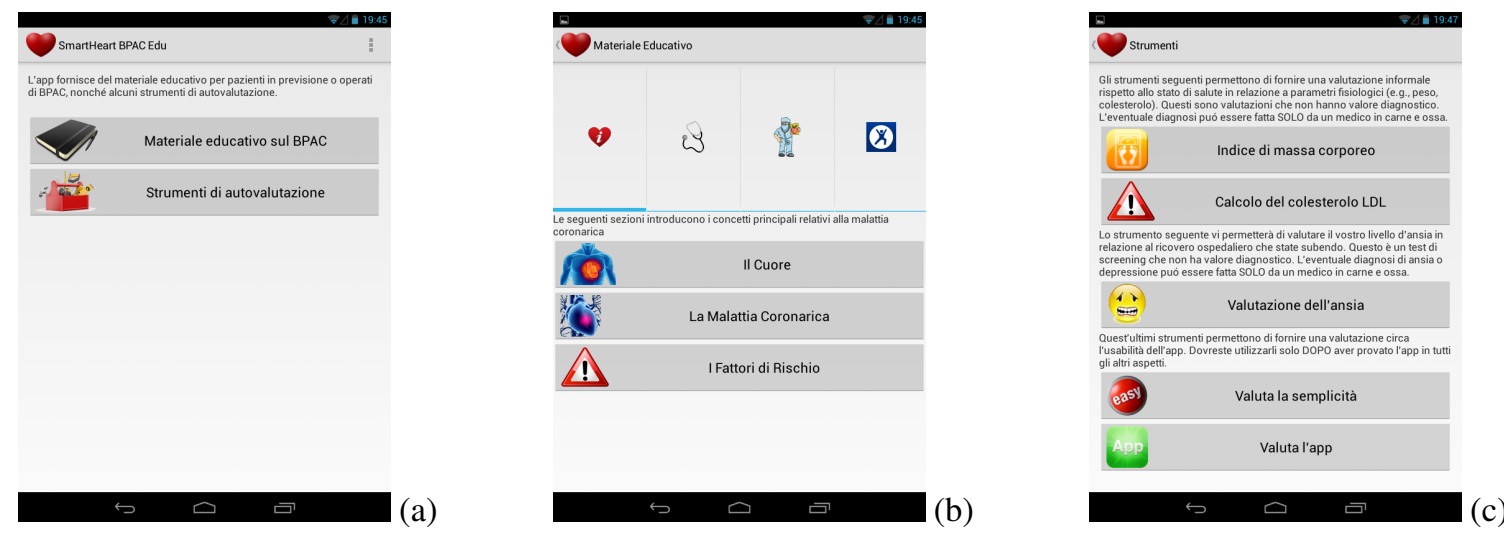

Figure 1: (a) App's home page showing both sections available, (b) educational material divided into four subsections indicated by clipart icons, (c) self-assessment tools page

disease experience intense emotions such as depression, aggression, anxiety, frustration and fear which can cause them to behave irrationally. (Gallagher and McKinley, 2007) report that patients who experience anxiety before a CABG have more post-operative pain, less long-term relief of cardiac signs and symptoms, more re-admissions, poorer quality of life and worse long-term psychological outcomes. The effect of pre-operative education in CABG is still debated: (Rahimianfar et al., 2013) concluded that patient education before CABG surgery is able to reduce both patient's anxiety and the need for sedatives; on the contrary, (Shuldham et al., 2002) demonstrate that there is no benefit to be gained from pre-operative education.

In such a context, the SmartHeart CABG Edu app was conceived: it is meant to be an innovative and up-to-date tool for patient education, the first of its kind in the Italian context. The SmartHeart CABG Edu is therefore an app that was developed to provide educational material for patients about to undergo CABG surgery and a set of self-assessment tools concerning both their health status, i.e., BMI calculator (Garrow and Webster, 1984), LDL cholesterol calculator (Friedewald et al., 1972) and anxiety assessment tool (Zigmond and Snaith, 1983), as well as their experience with the app, i.e., Single Ease Question (SEQ) (Sauro and Dumas, 2009) and System Usability Scale (SUS) (Brooke, 1996). The app runs on Android, was developed using Android Studio, $2.2 \mathrm{Mb}$ in size and does not require Internet to access the educational resources.

\section{First Prototype}

The first prototype of the app was developed at the end of 2014. The home page is made up of two sections (Fig. 1-a), namely: (i) the educational material, (ii) the self-assessment and usability tools. Tapping on the educational material button, the user is taken to the educational content, organized in four subsections: (i) anatomy of the heart and CAD info, (ii) diagnostic tests and therapeutic procedures, (iii) CABG surgery, cardiac rehab and (iv) healthy lifestyles (Fig. 1-b). Tapping on the self-assessment tools button, the user can use: the BMI and the LDL cholesterol calculators, the anxiety assessment tool and the two usability evaluation tools (Fig. 1-c). It is worth remarking that the self-assessment tools can be used by the patient to check whether he/she is actually following the healthy lifestyles described in the educational material, i.e., a normal weight through the BMI calculator, a reduced fat intake through the LDL cholesterol calculator.

Regular Issue

Vol 4 n. 1

http://adcaij.usal.es
Advances in Distributed Computing and Artificial Intelligence Journal 


\section{Evaluation}

The choice of performing one type of evaluation over another has to be established in relation to the stage of the project, what to evaluate, the available experts, as well as the time constraints and the available resources of the project (Nielsen and Mack, 1994). Since our project was in its first release, coherently with the state of the art, we decided (i) to ask two usability experts for an heuristic evaluation to generate large numbers of potential usability problems (Subsect. 3.1); (ii) to inquire primary stakeholders, i.e., patients (Subsect. 3.2). Finally, we investigated on the pre-operative anxiety of patients, before or after their reading the educational material (Subsect. 3.3). Note that the results reported below are an improved and updated version of the preliminary evaluations reported in (Di Giammarco et al., 2015).

\subsection{Expert-based review}

The most popular type of expert evaluation is the heuristic evaluation (Nielsen and Mack, 1994). Two evaluators were enrolled for the expert-based review: they used a new check list specifically designed to evaluate mobile interfaces, that reuses $69 \%$ of literature heuristics, the rest deriving from best-practices and recommendations for mobile interfaces (Gómez et al., 2014). Nevertheless, given that the app is a first prototype, the heuristics used for the evaluation were limited to only those actually applicable. Accordingly, Tab. 1 summarizes the expert-evaluation results: the table lists the items evaluated and the corresponding rating, i.e., a numeric value (Likert scale), for the two experts $E_{1}$ and $E_{2}$, where

0 means very poor usability,

1 means poor usability,

2 means sufficiently usable,

3 means good usability, and

4 means very good usability.

Regular Issue

Vol 4 n.1

http://adcaij.usal.es
Advances in Distributed

Computing and Artificial Intelligence Journal 


\begin{tabular}{|c|c|c|}
\hline \multirow{2}{*}{ Item } & \multicolumn{2}{|c|}{ Rating } \\
\hline & $E_{1}$ & $\mathrm{E}_{2}$ \\
\hline \multicolumn{3}{|c|}{ Visibility of system status } \\
\hline System status feedback & 3 & 3 \\
\hline Response time & 4 & 4 \\
\hline Selection/input of data & 2 & 3 \\
\hline Presentation adaptation & 0 & 0 \\
\hline \multicolumn{3}{|c|}{ Match between system and the real world } \\
\hline Metaphors/mental models & 2 & 1 \\
\hline Navigational structure & 1 & 1 \\
\hline Menus & 1 & 1 \\
\hline Simplicity & 2 & 3 \\
\hline Output of numeric information & 4 & 4 \\
\hline \multicolumn{3}{|l|}{ User control } \\
\hline Explorable interfaces & 2 & 3 \\
\hline Some level of personalization & 0 & 0 \\
\hline Process confirmation & 2 & 1 \\
\hline Undo/cancellation & 2 & 1 \\
\hline Menus control & 2 & 1 \\
\hline \multicolumn{3}{|l|}{ Consistency } \\
\hline Design consistency & 3 & 3 \\
\hline Naming convention consistency & 2 & 3 \\
\hline Menus/task consistency & 3 & 4 \\
\hline Functional goals consistency & 3 & 3 \\
\hline System response consistency & 2 & 3 \\
\hline Orientation & 4 & 4 \\
\hline \multicolumn{3}{|c|}{ Flexibility and efficiency of use } \\
\hline Search & 0 & 0 \\
\hline Navigation & 2 & 3 \\
\hline \multicolumn{3}{|c|}{ Aesthetic and minimalist design } \\
\hline Multimedia content & 1 & 2 \\
\hline Icons & 2 & 2 \\
\hline Menus & 1 & 2 \\
\hline Orientation & 3 & 3 \\
\hline Navigation & 1 & 2 \\
\hline
\end{tabular}

Table 1: Expert-based evaluation result

Regular Issue

Vol 4 n.1

http://adcaij.usal.es
Advances in Distributed

Computing and Artificial Intelligence Journal 
The agreement between the ratings given by the two experts was measured through a weighted Cohen's Kappa (Cohen, 1968). The estimated agreement was 0.82 , with 95\% confidence intervals of [0.70,0.94]. Accordingly, given the high rate of agreement between the two experts, the results summarized below can be considered reliable.

The median rating is sufficiently usable, the inter-quartile range (IQR) of two categories, and the $95 \%$ confidence intervals are [sufficiently usable, good usability]. As known, median and IQR are the most proper indexes for central tendency and dispersion for categorical variables, while confidence intervals reports what we might expect by other evaluations. Accordingly, the app can be considered sufficiently usable, the ratings have a large dispersion, and we might expect - by enrolling further evaluators - even a median rating of good usability.

Few suggestions about how to improve usability follow. The app should be improved in adaptability (e.g. the buttons' dimensions) and in the user personalization (e.g., an elderly person might have problems with font size). The contents should be supported via multimedia, in order to justify a technological support. Visual design might be improved (e.g., using a coherent color palette for icons). The affordance of browsing and navigation might be improved by introducing a menu (e.g., search the educational material). Furthermore, the auto-evaluation questionnaires' layout should be presented using boxes in order to improve the text comprehension. Finally, the health and usability questionnaires should be placed in separate sections, since they pursue different aims.

\subsection{User experience}

For investigating the user experience, we involved both primary and secondary stakeholders. The primary stakeholders are patients that have already undergone or are about to undergo CABG surgery. The secondary stakeholders are the health-care workers of the involved hospital wards (e.g., critical care unit, cardiac surgery). The paper reports only on the results coming from the primary stakeholders: for those regarding the secondary stakeholders (which were not involved in the improved evaluation reported in the paper), see (Di Giammarco et al., 2015).

The evaluation with the primary stakeholders was carried out from the 23rd of January, 2015 to the 27th of May, 2015. Before the observational sessions, the evaluator explained the goals of the evaluation to the patients, using the guidelines for an elderly observational evaluation where necessary (i.e., speaking aloud and slowly, using easy vocabulary and describing the goal using a family approach, to make sure all patients understood perfectly). A friendly environment was established. The focus groups took place after the observational evaluation sessions.

We gathered:

- qualitative data, as annotated by the evaluators, and

- quantitative data regarding the user experience, reported through the SEQ (Sauro and Dumas, 2009), SUS (Brooke, 1996), and with the Time-On-Task (Seffah et al., 2006).

As known, the Single Ease Question (SEQ) is a 7-point rating scale that is used to assess how a task was found easy to accomplish by users. The higher the value of the response, the easier the task is. We asked to rate the three tasks of:

Regular Issue

Vol 4 n.1

http://adcaij.usal.es
Advances in Distributed

Computing and Artificial Intelligence Journal 
(T1) reading the educational material,

(T2) using the BMI calculator, and

(T3) using the LDL cholesterol calculator.

The System Usability Scale (SUS) instead is a reliable tool for measuring usability, consisting of a 10 item questionnaire with five response options per item. It can be used to evaluate a wide variety of products and services, including mobile apps. The tool rates a system with a score ranging from 0 to 100 . The higher the score, the more usable the system is. For a simpler interpretation of the SUS score, we introduced a qualitative interpretation, based on the thresholds computed in (Bangor et al., 2009), that "converts" the score into the seven adjectives for the app, namely: worst imaginable, awful, poor, OK, good, excellent, best imaginable.

Finally, the Time-On-Task (ToT) is the total time taken by a user to complete a task.

A total of 15 patients were involved in the study, seven coming from the Coronary Unit of Pescara Hospital, five from the Neurosurgery Unit of Chieti Hospital, three from the Cardiology Unit of Avezzano Hospital. Not all patients completed the SEQ and SUS questionnaires, but for all of them we could analyze the ToT data. The results follow.

\subsubsection{Quantitative data}

SEQ The distribution of the SEQ scores is reported in Fig. 2. In terms of central tendency, the median for all tasks is "easy". Therefore, on average, no particular difficulties were reported, even if few users rated the tasks below the sufficiency.

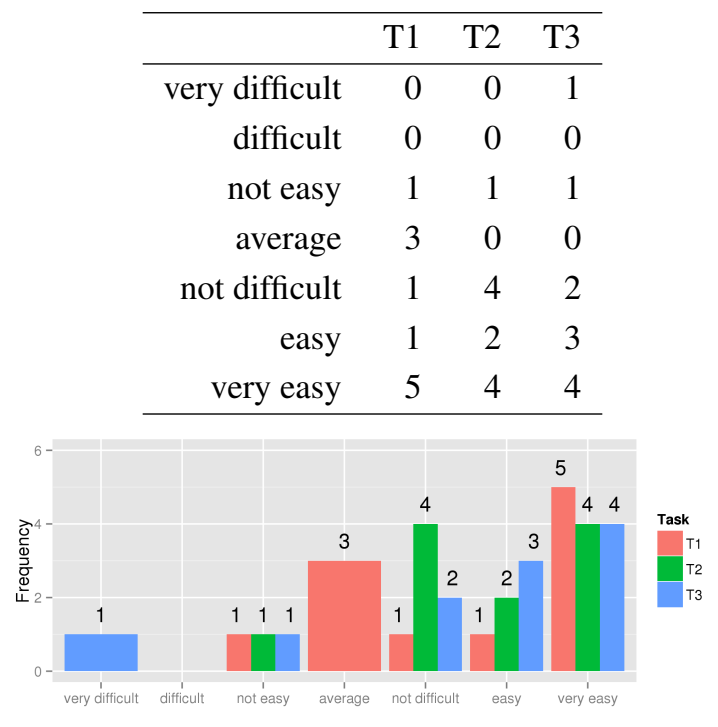

Figure 2: Descriptive analysis of the SEQ score

SUS The distribution of the SUS score is shown in Fig. 3. The median rating is $O K$, even if we found one rating as best imaginable, two other ratings as good, versus one poor rating.

To investigate further into the SUS score, we analyzed the answers to each question (Tab. 2). There are no questions with clearly negative answers. However, there are three questions with discordant answers, i.e.,

Regular Issue

Vol 4 n.1

http://adcaij.usal.es
Advances in Distributed

Computing and Artificial Intelligence Journal 


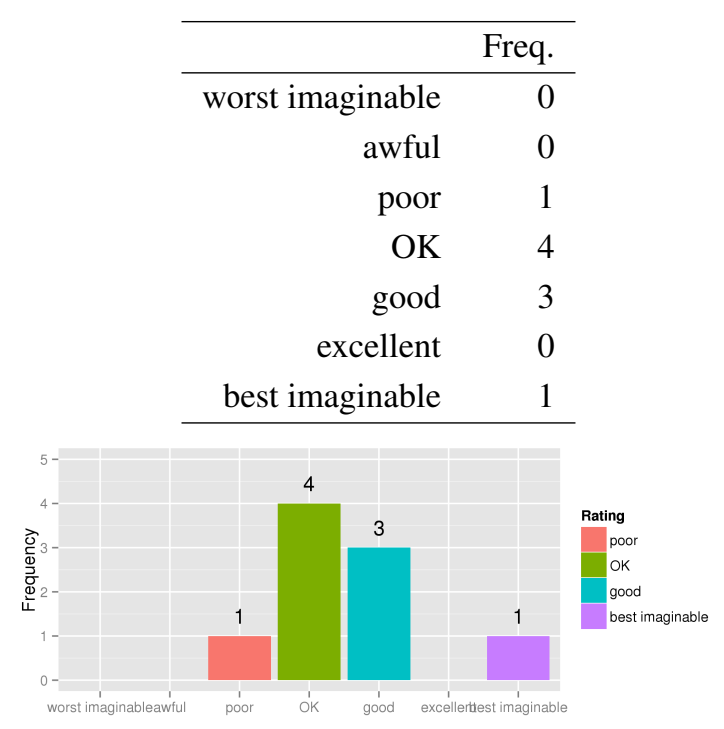

Figure 3: Descriptive analysis of the SUS scores

\begin{tabular}{rrrrrrrrrrr}
\hline & Q1 & Q2 & Q3 & Q4 & Q5 & Q6 & Q7 & Q8 & Q9 & Q10 \\
\hline strongly disagree & 1 & 5 & 0 & 3 & 0 & 6 & 0 & 5 & 0 & 3 \\
disagree & 1 & 2 & 0 & 1 & 0 & 2 & 0 & 2 & 2 & 1 \\
average & 3 & 1 & 2 & 2 & 2 & 0 & 2 & 1 & 3 & 1 \\
agree & 1 & 1 & 5 & 1 & 4 & 1 & 5 & 1 & 1 & 3 \\
strongly agree & 3 & 0 & 2 & 2 & 3 & 0 & 2 & 0 & 3 & 1 \\
\hline
\end{tabular}

Table 2: Descriptive analysis of the SUS score by question

questions "Q1" (I think that I would like to use this system frequently), "Q4" (I think that I would need the support of a technical person) and "Q10" (I needed to learn a lot of things to use this system).

As for "Q1", the reason is that the app was developed especially for the pre-operative phase, and not for daily use. The results regarding "Q4" and "Q10" are instead more interesting and can be explained by the fact that, since the patients were elderly people, they were not accustomed to the use of smartphones.

The more positive aspects were in connection with "Q3" (I thought the system was easy to use), "Q5" (I found the various functions in this system were well integrated) and "Q7" (Most people would learn to use this system very quickly).

ToT The results of the descriptive analysis, for the Time On Task metric, are listed in Tab. 3. The first column lists all pages of the app, the second column lists the (average) seconds spent by the users on such a page before moving to another page.

Not surprisingly, the longest questionnaires (i.e., Anxiety and SUS) took longer times to be completed.

Furthermore, a very high average time (circa 3 minutes) is reported for the LDL calculator, probably because it needs to input numbers related to blood analyses that - even if common - requires a memory effort from the user or even to read the lab report.

Regular Issue

Vol 4 n.1

http://adcaij.usal.es
Advances in Distributed Computing and Artificial Intelligence Journal 
Finally, no particular problems are highlighted for the other pages.

\begin{tabular}{llr}
\hline & Task & Mean \\
\hline 1 & Anxiety & 121.78 \\
2 & BMI & 29.51 \\
3 & LDL calculator & 189.50 \\
4 & SEQ & 98.65 \\
5 & Ed.Mat. Page & 72.24 \\
6 & Ed.Mat. Index & 12.79 \\
7 & Main & 26.26 \\
8 & SUS & 243.03 \\
9 & Tools & 69.05 \\
\hline
\end{tabular}

Table 3: ToT descriptive analysis

\subsubsection{Qualitative data}

No user asked for support in browsing the GUI. However, in some cases, to go back from a page to the hierarchical higher, users took some time before using either the Android "back" button or tapping the "back" sign close to the activity title. Users also asked about the possibility of having the educational material in paper format: it is natural given that the users were elderly people. Finally, no particular problems emerged while calculating the body mass index and the LDL cholesterol. In all cases, improvements in terms of a bigger font size and button affordance (e.g., by adding text together with icons) was considered as required.

The focus groups confirmed some issues carried out in the observational evaluation phase, such as the font size issue, but also highlighted the enthusiasm of users in reading the educational material. One user thanked the evaluator for having provided him with educational material on CABG surgery, another asked for a paper copy to bring to her own cousin (so to better explain her problems). Furthermore, users suggested to improve on the multimediality of the material (e.g., by adding videos). A final remark is on the personalization of the app: users appreciated the possibility to calculate their own data and asked for more functions supporting their personal health status, e.g., storing their blood pressure values, alarms that reminded medication-intake time.

\subsection{Pre-operative anxiety}

For the analysis of the anxiety level, we submitted the Hospital Anxiety and Depression Scale (HADS) (Zigmond and Snaith, 1983), specifically developed to detect and manage the emotional disorder in patients under investigation and treatment in medical and surgical ward. To all patients enrolled in the evaluation we randomly asked to fill the questionnaire either before or after having read the educational material. Fig. 4 summarizes the descriptive analysis of the data: on the top the frequency table, on the bottom the corresponding histograms.

No patients reported significant anxiety levels after having read the educational material, vs two that instead showed a probable anxious status and five that did not reveal anxiety. Such a difference was not statistically significant (Wilcoxon rank-sum test, $p=0.1413$ ).

Regular Issue

Vol 4 n.1

http://adcaij.usal.es
Advances in Distributed Computing and Artificial Intelligence Journal 


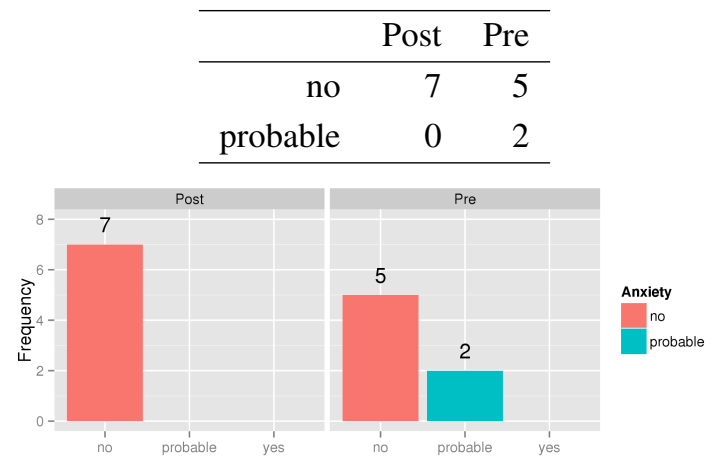

Figure 4: Descriptive analysis of the anxiety levels

\section{New prototype}

As for usability, the evaluations coming from the expert concluded that the user interface appears sufficiently usable and mentioned several points that can be improved. The primary stakeholders did not reported major usability problems with the app, but highlighted - both via quantitative and qualitative data - some improvements.

Accordingly, we enhanced the app in connection with the following points:

1. improved visual design, using a coherent color palette for icons (see $\$ 3.1$ );

2. a menu was introduced (see $\$ 3.1)$;

3. health and usability questionnaires were placed in two separated sections (see $§ 3.1$ );

4. an help dialog was added to tackle the issues reported by the two SUS questions "I think that I would need the support of a technical person" and "I needed to learn a lot of things to use this system". The help dialog is shown the first time a user shows a page, or every time is needed through the menu (see $\S 3.2 .1)$;

5. improved navigation in terms of explicit "back" buttons (see §3.2.2);

6. improved font size in the educational material (see $\S 3.1$ and $\S 3.2 .2$ );

7. the comprehension of the questionnaires was improved by adding boxes around the questions (see $\S 3.1)$.

Fig. 5 shows a sequence of snapshots of the latest version of the app, that can be compared with those reported in Fig. 1 to clearly view all introduced changes.

Fig. 5(a) depicts the main page of the app. It is now made up of three sections: the first for the educational material, the second for the self-assessment tools, the third for usability. All icons now follows the same visual design (refer to points 1, 2 and 3 ).

Fig. 5(b) shows the help page opened in the main page of the educational material. A "back" button is also present: it is below the list of topics, and can be glimpsed right behind the help dialog (refer to points 4 and 5 above).

Regular Issue

Vol 4 n.1

http://adcaij.usal.es
Advances in Distributed

Computing and Artificial Intelligence Journal 

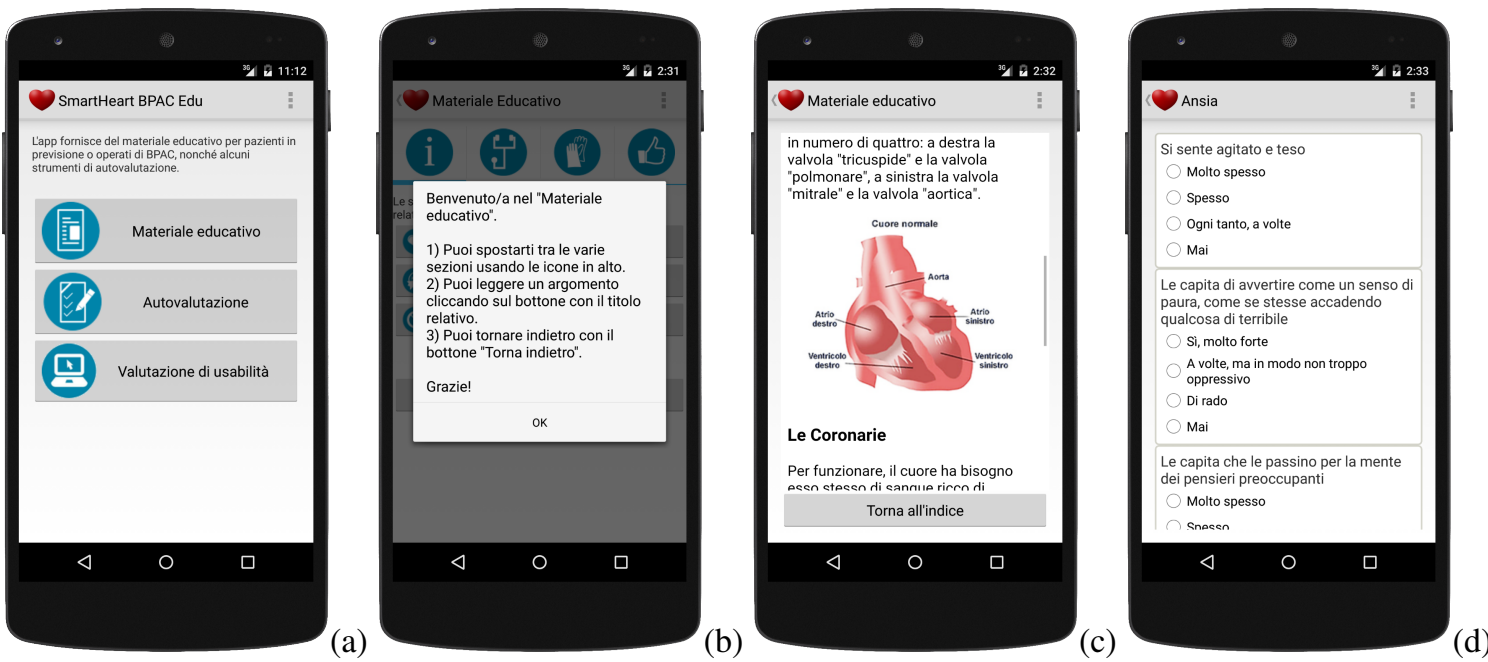

Figure 5: Snapshots of the latest version of the SmartHeart CABG Edu app

Fig. 5(c) shows how a topic is presented to a patient. A "back" button is present, and the font size has been increased (refer to points 5 and 6 above).

Fig. 5(d) depicts the questionnaire regarding anxiety. All questions have been "encapsulated" in boxes so improve the comprehension (refer to point 7 above).

\section{Conclusions}

The paper reported on the first prototype of an Android app developed for providing educational material to patients about to undergo CABG surgery, its evaluation from the twofold perspective of usability and health outcomes, and the improvements put into practice that lead to the current version.

In particular, starting from the first prototype, two experts were asked to report on the most important usability issues. Furthermore, fifteen patients were enrolled to test the app usability and the desirable outcome of reducing the pre-operative anxiety.

As for usability, by following the main suggestions coming from the experts, and a careful analysis of the SEQ and SUS questionnaires, along with the ToT data, enabled us to define a set of seven improvements. By following these suggestions, a new version of the application was developed and released.

In terms of anxiety, we could not find a statistically significant difference between patients that read and didn't read the educational material. However, given the reduced number of enrolled patients and the p-value close to the significance, we can still consider the result as encouraging.

Our future work will follow a twofold pathway. Firstly, by continuing with the remaining usability improvements connected with adaptation and personalization. Secondly, by extending the experiment regarding anxiety by enrolling a larger sample of patients: in such respect, the possibility of adopting a multi-center trial in which the SmartHeart CABG Edu will be used in more than one medical center or clinic is currently under discussion.

Regular Issue

Vol 4 n.1

http://adcaij.usal.es
Advances in Distributed

Computing and Artificial Intelligence Journal 


\section{Appendix A}

The web page:

http://vittorini.univaq.it/smart-heart

contains summary information about the SmartHeart CABG Edu app, as well as the possibility to download both the source code and the Android package.

Acknowledgment We would thank the personnel working in the Coronary Unit of the Pescara Hospital, in the Neurosurgery of Chieti Hospital and in the Cardiology Unit of the Avezzano Hospital for their cooperation.

\section{References}

Bangor, A., Kortum, P., and Miller, J., 2009. Determining What Individual SUS Scores Mean: Adding an Adjective Rating Scale. JUS - The Journal of Usability Studies, 4(3):114-123.

Black, J. M. and Hawks, J. H., 2009. Medical-Surgical Nursing: Clinical Management for Positive Outcomes. ISBN 978-1-4160-4687-5.

Brooke, J., 1996. SUS-A quick and dirty usability scale. Usability evaluation in industry, 189:194.

Cohen, J., 1968. Weighted kappa: Nominal scale agreement provision for scaled disagreement or partial credit. Psychological Bulletin, 70(4):213-220. ISSN 1939-1455(Electronic);0033-2909(Print). doi: $10.1037 / \mathrm{h} 0026256$.

Di Giammarco, G., Di Mascio, T., Di Mauro, M., Tarquinio, A., and Vittorini, P., 2015. SmartHeart CABG Edu: First Prototype and Preliminary Evaluation. In Methodologies and Intelligent Systems for Technology Enhanced Learning, number 374 in Advances in Intelligent Systems and Computing, pages 57-66. Springer International Publishing. ISBN 978-3-319-19631-2, 978-3-319-19632-9.

Friedewald, W. T., Levy, R. I., and Fredrickson, D. S., 1972. Estimation of the concentration of low-density lipoprotein cholesterol in plasma, without use of the preparative ultracentrifuge. Clinical chemistry, 18(6):499-502.

Gallagher, R. and McKinley, S., 2007. Stressors and anxiety in patients undergoing coronary artery bypass surgery. American Journal of Critical Care, 16(3):248-257.

Garrow, J. S. and Webster, J., 1984. Quetelet's index (W/H2) as a measure of fatness. International journal of obesity, 9(2):147-153.

Gómez, R. Y., Cascado Caballero, D., and Sevillano, J.-L., 2014. Heuristic Evaluation on Mobile Interfaces: A New Checklist. The Scientific World Journal, 2014:e434326. ISSN 2356-6140. doi:10.1155/2014/ 434326.

Nielsen, J. and Mack, R. L., 1994. Usability Inspection Methods. John Wiley \& Sons Inc, New York. ISBN 9780471018773.

Rahimianfar, A. A., Javadi, S. S., Dehghani, H., Sareban, M. T., Akbarzade, T., Eslami, A., Rahimianfar, F., and Khosravi, A., 2013. The effect of Training Booklet on anxiety level of the patients candidate for coronary artery bypass graft surgery. Journal of Biology and today's world, 2(10).

Sauro, J. and Dumas, J. S., 2009. Comparison of three one-question, post-task usability questionnaires. In

Regular Issue

Vol 4 n.1

http://adcaij.usal.es
Advances in Distributed

Computing and Artificial Intelligence Journal 
Proceedings of the SIGCHI Conference on Human Factors in Computing Systems, pages 1599-1608. ACM.

Seffah, A., Donyaee, M., Kline, R. B., and Padda, H. K., 2006. Usability measurement and metrics: A consolidated model. Software Quality Journal, 14(2):159-178.

Shuldham, C. M., Fleming, S., and Goodman, H., 2002. The impact of pre-operative education on recovery following coronary artery bypass surgery. A randomized controlled clinical trial. European heart journal, 23(8):666-674.

Young, A., Geyer, N., and Young, A., 2009. Juta's manual of nursing. v. 1. Juta, Limited. ISBN 9780702180323.

Zigmond, A. S. and Snaith, R. P., 1983. The hospital anxiety and depression scale. Acta psychiatrica scandinavica, 67(6):361-370.

Regular Issue

Vol 4 n. 1

http://adcaij.usal.es
Advances in Distributed

Computing and Artificial Intelligence Journal 